\title{
Pregnancy-triggered triple autoimmunity (Hashimoto's thyroiditis, antiphospholipid syndrome and systemic lupus erythematosus)
}

\author{
Shoaib Z Junejo ${ }^{1 *}$, Sandeep Singh Lubana¹, Adriana Abrudescu-Opran², Issac Sachmechi ${ }^{3}$
}

\begin{abstract}
${ }^{*}$ Correspondence
Shoaib Z Junejo

Department of Medicine Icahn School

Of Medicine at Mount Sinai/NYC

Health+Hospital/Queens, New York,

USA

Email: shoaibjunejo88@gmail.com

'Department of Medicine Icahn School Of Medicine at Mount Sinai/ NYC

Health + Hospital/ Queens, New York, USA

2Department of Rheumatology Icahn School Of Medicine at Mount Sinai/ NYC Health + Hospital/ Queens, New York, USA

3Department of Endocrinology Icahn School Of Medicine at Mount Sinai/ NYC Health + Hospital/ Queens, New York, USA
\end{abstract}

Received: Apr 16, 2018

Accepted: May 23, 2018

Published: May 30, 2018

\begin{abstract}
Introduction: We describe a case of 22 year old female with her first pregnancy triggered Hashimoto's thyroiditis (HT), Antiphospholipid Syndrome (APS) and Systemic Lupus Erythematosus (SLE).
\end{abstract}

Case Report: A 22 year old female was diagnosed with HT on levothyroxine during the early first trimester. During 21 weeks of gestation patient has intrauterine fetal demise and underwent medical abortion. SLE work up including antinuclear antibody and anti-double stranded DNA were positive. She underwent kidney biopsy, which revealed membranous and mesangial proliferative lupus nephritis. Diagnosis of SLE and APS waS made. Treatment with anticoagulation therapy was started. SLE therapy was initiated with prednisone, mycophenolate mofetil and hydroxychloroquine with complete resolution of symptoms.We report a 23-year-old gravida in her first pregnancy, suffering from MGN and severe nephrotic syndrome, complicated by APLA syndrome. The patient was treated with enoxaparin, aspirin azathioprine, and Prednisone for a short time, in addition to furosemide and albumin intravenously. She was delivered at 30 weeks due to deteriorating maternal and foetal conditions.

Discussion: APS is a prothrombotic disorder with various manifestations, most commonly venous and arterial thromboembolism and recurrent pregnancy loss. Pregnancy may trigger an underlying APS, which may well be the causative for the miscarriage. New onset SLE during pregnancy is rare. However, in our case, the anemia, thrombocytopenia, and proteinuria led us to the correct diagnosis of SLE. HT is associated with higher rates of infertility and early miscarriages, due to the associated hormonal changes and instability. However, the association of APS and HT is not well recognized in pregnant women.

Conclusion: We present here a challenging case of new-onset triple autoimmune disorders trigged by pregnancy. Clinicians should be aware of this association and initiate early autoimmune work up for SLE and APS in patients with new onset of HT during pregnancy.

A successful neonatal and maternal outcome was achieved in this case. The patient's history revealed thrombocytopenia and APLA syndrome and continues to be treated chronically with enoxaparin. Kidney biopsy performed after delivery showed membranous MGN stage II-III. Herein, we present a case of successful pregnancy and foetal outcome in a young woman with APLA syndrome and MN.

Keywords: Pregnancy triggered Hashimoto's thyroiditis, Antiphospholipid Syndrome and Systemic Lupus Erythematosus 


\section{Introduction}

Although the association between autoimmune thyroid and rheumatic disorders has been studied in nonpregnant women and there are no data on the frequency of this association during pregnancy and its impact on reproductive outcomes. We present a case of 22 year old female with her first pregnancy triggered Hashimoto's thyroiditis (HT), Antiphospholipid Syndrome (APS) and Systemic Lupus Erythematosus (SLE).

\section{Case Report}

A 22 year old female diagnosed with HT during the early first trimester, Her thyroid work up include antithyroid peroxidase antibody was positive, she was stated on levothyroxine. She was admitted at 21 weeks of gestation for labor induction secondary to intrauterine fetal demise and underwent medical abortion. Laboratory results was significant for thrombocytopenia, prolongation activated partial thromboplastin time, positive IgG and IgM anticardioloipin antibodies, anti-beta2- glycoprotein I and lupus anticoagulant. Placental pathology showed placental infarcts with hypoxia ischemic changes. Due to suspicion of APS and therefore risk of thromboembolism, the patient was started on prophylactic Lovenox 40mg SC daily. She presented to the emergency room 4 weeks later with sudden onset of focal neurologic deficit. Computerized tomography angiogram showed distal right middle cerebral artery segment M1 occlusion. Patient was started on therapeutic anticoagulation and focal weakness was resolved in 5 days. SLE work up initiated, antinuclear antibody and anti-double stranded DNA were positive. Anti-smith antibody, anti-RNP antibody, anti-Ro, anti-La antibodies were reported negative with normal $\mathrm{C} 3$ and $\mathrm{C} 4$ complement levels. $24 \mathrm{hr}$ urine protein was between 1.56 and $2 \mathrm{gm}$. She underwent kidney biopsy, which revealed membranous and mesangial proliferative lupus nephritis, ISA/RPS class V and II. Diagnosis of SLE and APS was made. Treatment with anticoagulation therapy was started with warfarin and aspirin. SLE therapy was initiated with prednisone, mycophenolate mofetil and hydroxychloroquine with complete resolution of proteinuria. For HT levothyroxine was adjusted.

\section{Discussion}

APS is a prothrombotic disorder with various manifestations, most commonly venous and arterial thromboembolism and recurrent pregnancy loss. Diagnosis of APS can be challenging due to evolving criteria and overlapping characteristics with other prothrombotic thrombocytopenic disorders. Thrombotic complications within the uteroplacental circulation has also been proposed as a contributing mechanism. Pregnancy may trigger an underlying APS, which may well be the causative for the miscarriage [1-3] , In our case given the placental findings the fetal death was due to APS. New onset SLE during pregnancy is rare. However, in our case, the anemia, thrombocytopenia, and proteinuria led us to the correct diagnosis of SLE. When SLE is first suspected during pregnancy, the diagnostic criteria are not different from those for nonpregnant women. Renal disorders appeared to be more common at the onset of SLE in pregnant patients than in nonpregnant patients. Meanwhile, HT is associated with higher rates of infertility and early miscarriages, due to the associated hormonal changes and instability. However, the association of APS and HT is not well recognized in pregnant women [4-7] ].

\section{Conclusion}

We present here a challenging case of new-onset triple autoimmune disorders trigged by pregnancy. Our case confirms a close association between autoimmune thyroiditis, SLE and APS during pregnancy. Clinicians should be aware of this association and initiate early autoimmune work up for SLE and APS in patients with new onset of HT during pregnancy.

\section{Copyrights}

(c) Shoaib Z Junejo et al, 2018; licensee OA Journal of Clinical Case Reports. This is an Open Access article distributed under the terms of the Creative Commons Attribution License (http:// creativecommons.org/licenses/by/4.0), which permits unrestricted use, distribution, and reproduction in any medium, provided the original work is properly credited.

\section{References}

1. Hoayek JG, Moussa HN, Rehman HA, et al. (2016) Catastrophic antiphospholipid syndrome in pregnancy, a diagnosis that should not be missed. J Matern Fetal Neonatal Med. 29: 1-6.

2. Schreiber K (2016) Pregnancies in women with systemic lupus erythematosus and antiphospholipid antibodies. Lupus. 25 343-345.

3. Chaturvedi S, McCrae KR (2015) The antiphospholipid syndrome: still an enigma. Hematology Am Soc Hematol Educ Program. 2015: 53-60. 
4. de Jesus GR, Mendoza-Pinto C, de Jesus NR, et al. (2015) Understanding and Managing Pregnancy in Patients with Lupus. Autoimmune Dis. 2015: 943490.

5. LV J, Wang W, Li Y (2015) Clinical outcomes and predictors of fetal and maternal consequences of pregnancy in lupus nephritis patients. Int Urol Nephrol. 47: 1379-1385.
6. Buyon JP, Kim MY, Guerra MM, et al. (2015) Predictors of Pregnancy Outcomes in Patients With Lupus: A Cohort Study. Ann Intern Med. 163: 153-163.

7. Tomas NM, Beck LH, Meyer-Schwesinger C, et al. (2014) Thrombospondin type-1 domain-containing 7A in idiopathic membranous nephropathy. N Engl J Med 371: 2277-2287. 